\title{
INCONTINENCIA URINARIA DE ESFUERZO EN LA MUJER ESTABLECIMIENTO DE UNA NORMA DE TRATAMIENTO Y ESTUDIO CLINICO DE 28 CASOS
}

\author{
Dr. Pedro Nel Cardona* \\ Dr. Jaime Botero Uribe* \\ Dr. Alfonso Jubiz Hasbun* \\ Dr. Antonio Vélez Zapata* \\ Dr. René Díaz Correa* \\ Dr. Jaime Uribe Duque*
}

\section{Introducción}

Fue la mente siempre inquieta del profesor Pedro Nel Cardona la que agitó con insistencia este tema en las reuniones clínicas que se celebraron en el Departamento en las últimas semanas del año de 1967, justamente cuando nos preparábamos a asistir al VII Congreso de la especialidad, que se realizaría en Cúcuta.

El mismo propuso este tema como la contribución que haría el grupo de Antioquia al Congreso que ahora se celebra en esta ciudad de Manizales y suyo era el encargo de hacer la introducción al mismo, como justo reconocimiento por su labor pionera en este como en todos los campos en que espigó su vivaz inteligencia.

Sea pues este el momento de rendirle un justo tributo de admiración y de recalcar su eficaz colaboración en el estudio de las pacientes y en la compilación de los resultados, labores que realizó trabajando hombro a hombro con nosotros.
Desde Febrero de 1968 iniciamos la organización de una consulta dedicada al estudio de este tema y constituímos un grupo de trabajo con los profesores que voluntariamente quisieron colaborar en él. Como primer paso nos dimos a la tarea de revisar la literatura mundial y colombiana de los últimos años y nos encontramos en la primera dos tipos de artículos: uno que trataba de técnicas y procedimientos diversos para curar la incontinencia de esfuerzo, (1, 2, $3,4,5,6,7)$ y otro que se refería a estudios básicos sobre fisiología de la micción y sobre fisiopatología de la misma entidad. Los trabajos que nos parecieron resumir mejor los hallazgos más recientes y dar la mejor explicación a los cambios anatómicos, son los de Bailey (8), Jeffcoate (9), Hodgkinson (10, 11), y Green $(12,13)$.

Revisamos igualmente la literatura colombiana $y$ encontramos varios au-

* Del Departamento de Ginecología y Obstetricia de la Facultad de Medicina de la Universidad de Antioquia. 
tores que se han ocupado del tema aunque no muchos. Destacamos acá las contribuciones de Beltrán (14) y de Bernal (15).

\section{Definición}

Se entiende por incontinencia urinaria de esfuerzo en la mujer la pérdida súbita, involuntaria e intermitente de orina a través de una uretra intacta, bajo ciertas condiciones que causan un aumento en la presión intraabdominal (11). Es considerada por algunos autores (16) como una variedad de incontinencia urinaria intermitente.

\section{Fisiopatología y Anatomía patológica}

Se sabe actualmente que el problema básico en la incontinencia urinaria de esfuerzo es el de un soporte inadecuado de la base y cuello vesicales y de la uretra proximal, con el resultado de una distorsión específica y más bien característica en la anatomía uretrovesical. Como consecuencia de ello la uretra proximal, que normalmente es un órgano intra-abdominal, se desplaza y se coloca fuera del campo correspondiente de fuerza. Así, un aumento súbito de la presión intra-abdominal, que normalmente se transmite igualmente a la vejiga y a la uretra proximal y no desencadena salida de orina por ser mayor la presión en ésta que en aquélla, resulta en la pérdida de la orina por producirse un incremento de presión mayor en la vejiga que en la uretra.

El aspecto radiográfico de esta situación se aprecia claramente en la desaparición del ángulo uretro-vesical posterior y en el descenso rotacional de la uretra que hace que el ángulo de ella con la vertical, que normalmente es de 30 grados, aumente en forma variable (Fig. 1).
Parece que más importante aún que la colocación espacial de la base de la vejiga y de la uretra proximal, es la configuración anatómica uretrovesical mencionada, manifiesta en el ángulo uretro-vesical posterior, que debe ser normalmente de 90 a 100 grados, y en la relación del eje de la uretra con la vertical, que normalmente forma un ángulo de 30 grados. Como consecuencia de estas alteraciones, al ocurrir un esfuerzo la presión intra-abdominal es mayor en la vejiga que en la uretra y se produce la salida de orina.

Los estudios radiográficos pre y post-operatorios han confirmado este punto de vista al observarse que la corrección del ángulo uretro-vesical posterior va siempre acompañado de la corrección del trastorno funcional, es decir, la incontinencia de esfuerzo.

De acuerdo con los estudios de Bailey (8) y de Green (12) ya mencionados, los casos de incontinencia de esfuerzo grado l, en los cuales hay solamente pérdida del ángulo uretrovesical posterior, se mejoran fácilmente en un alto porcentaje con una intervención vaginal, mientras que aquellos con una incontinencia de esfuerzo grado II, en la cual tanto el ángulo uretro-vesical como el eje de la uretra estaban alterados, necesitan procedimientos quirúrgicos más complejos que en muchas ocasiones incluyen la vía vaginal y abdominal en forma simultánea.

\section{Objetivos}

Al iniciar la consulta de incontinencia urinaria nos propusimos adoptar una serie de normas para llegar al diagnóstico de la entidad; definir y llevar a cabo una serie de procedimientos quirúrgicos destinados a corregir las diferentes variedades diagmosticadas, y finalmente seguir el control de las pacientes por un lapso 


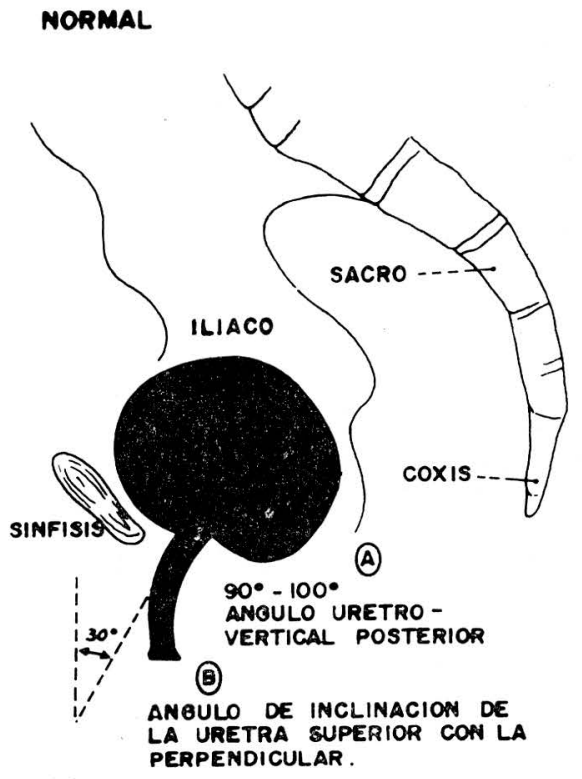

no menor de dos años para tratar de determinar el resultado a largo plazo.

\section{Métodos}

Las pacientes seleccionadas en forma preliminar en la consulta externa general, fueron sometidas al siguiente estudio diagnóstico:

1. Historia clínica completa, en la cual se averiguaba por antecedentes varios y muy particularmente por aquellos relacionados con la función vesical, tratando de determinar con la mayor precisión el tipo de trastorno miccional que presentaba la paciente.

2. Cuestionario sobre trastorno miccional. Se sometió a las pacientes

\section{INCONTINENCIA DE ESFUERZO}
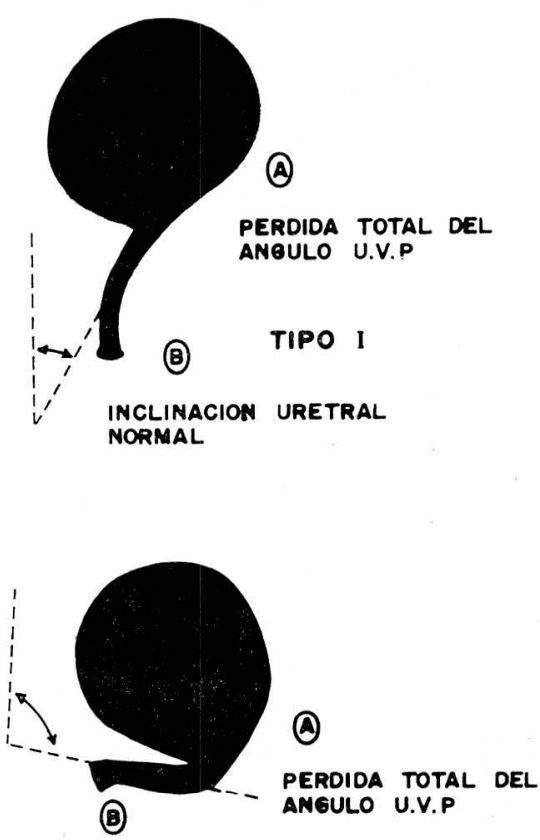

AUMENTO E INVERSION DEL

EJE HORMAL DE INCLIMACION URETRAL DEOIDO A DESCENGO ROTACIONAL DE LA URETRA.

TIPO

al cuestionario que presenta Hodgkinson (10), con algunas pequeñas modificaciones adjetivas.

3. Examen físico general completo y exámenes especiales de laboratorio: coprológico, hemoglobina, hematocrito, orina, leucograma y sedimentación.

4. Examen ginecológico completo, que se iniciaba pasando una sonda a la vejiga para medir residuo urinario y tomar muestras en frasco estéril para estudio bacteriológico.

5. Pruebas de incontinencia. Se introducían luego a la vejiga $250 \mathrm{cc}$. de agua estéril y se pedía a la paciente que hiciera esfuerzos de pujo, tanto en posición de litotomía como de 
pies. Se hacían a continuación las pruebas de Bonney y de Read, en ambas posiciones.

6. Estudio urológico. A algunas pacientes se les hacía a continuación un estudio urológico que incluía cistoscopia y uretroscopia para buscar inflamaciones, divertículos, tumores, etc. También se pedía interconsulta al urólogo en casos de encontrarse infección urinaria en el estudio de la orina tomada con sonda.

En unos pocos casos se hizo cistometría con la técnica descrita por
Mitchell (17), con el fin de determinar el tono vesical y sus variaciones y tratar de determinar las vejigas neurogénicas hipo o hipertónicas.

7. Estudio radiológico. Siempre que fue posible, se llevó a cabo un estudio radiológico según la técnica descrita por Hodgkinson y otros (18). Los hallazgos radiológicos de este estudio constituyeron el principal criterio que se tuvo en cuenta en la mayoría de los casos para la elección del procedimiento terapéutico, tal como más adelante se explica (Figs. 2, $3,4,5,6,7$ ).

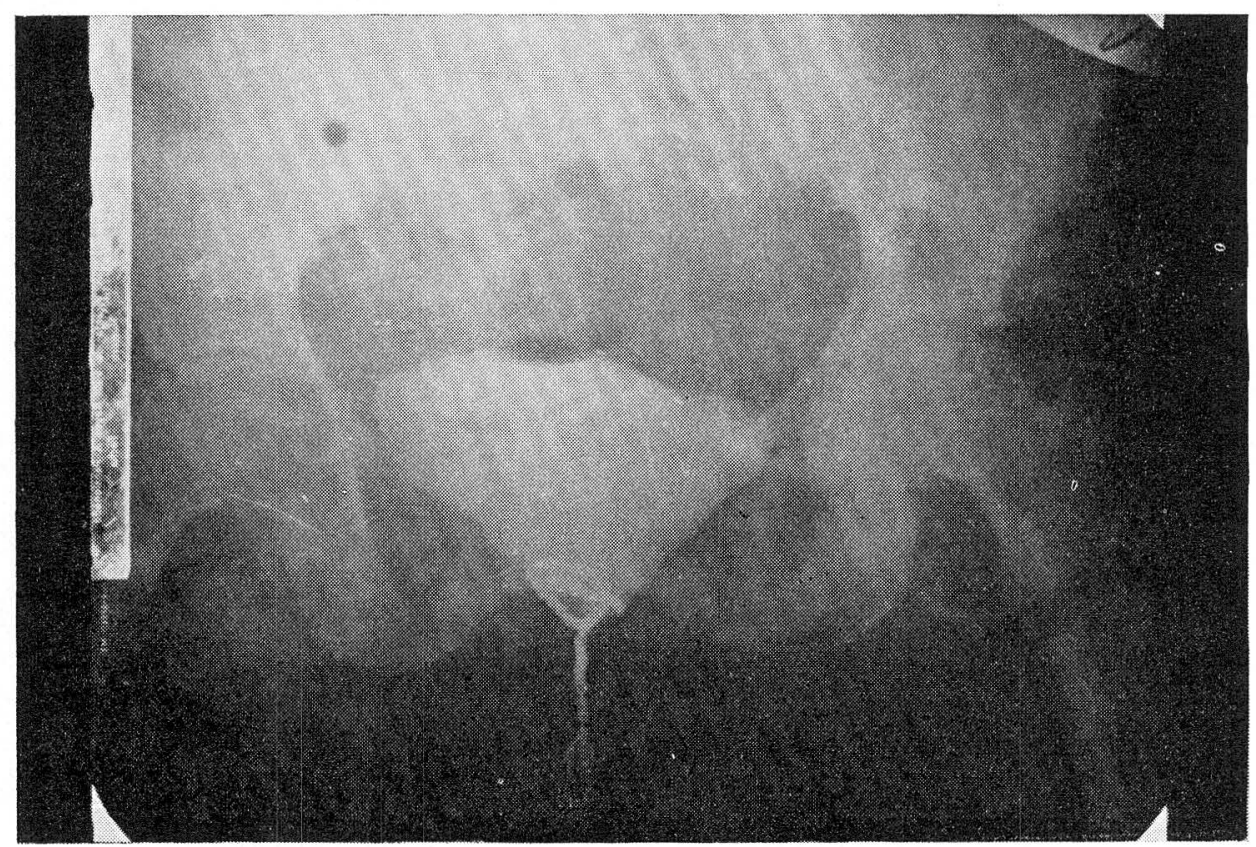

FIGURA 2 


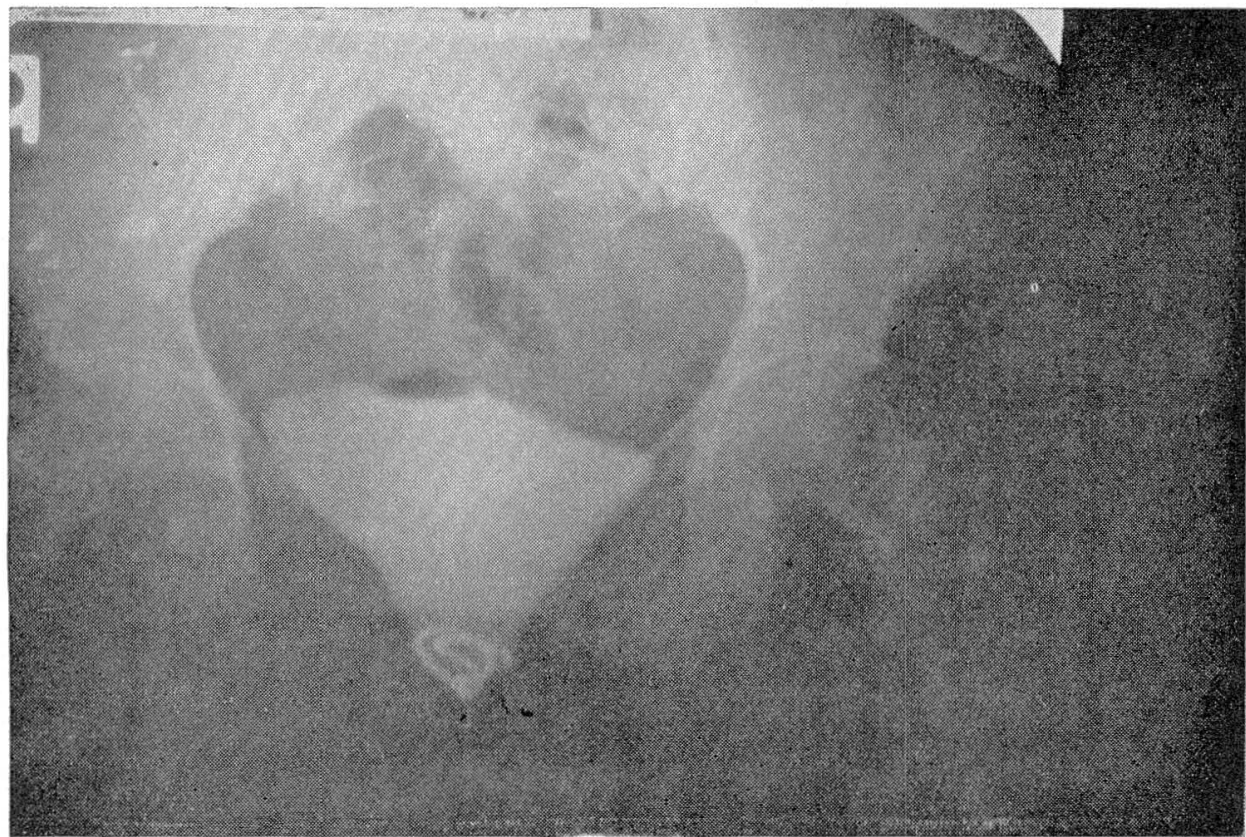

FIGURA 3

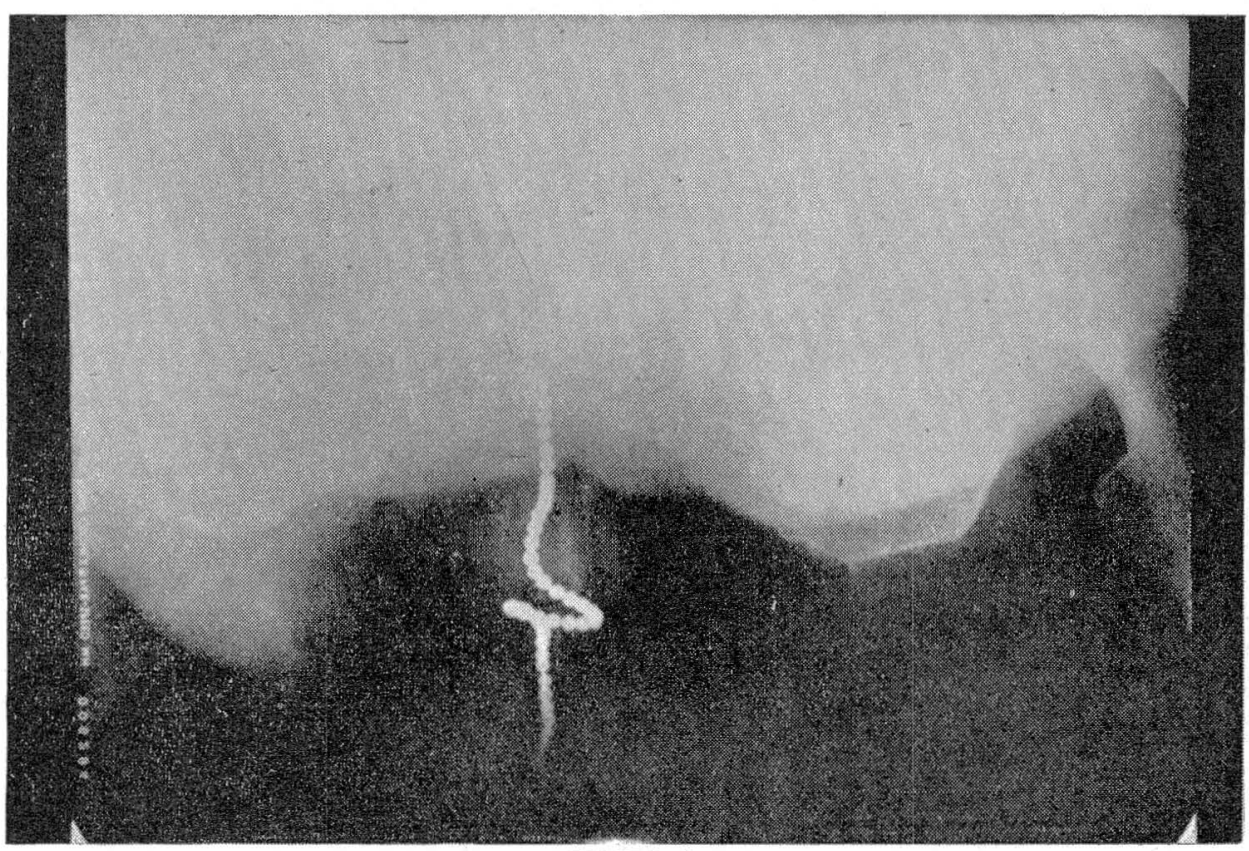

FIGURA 4 


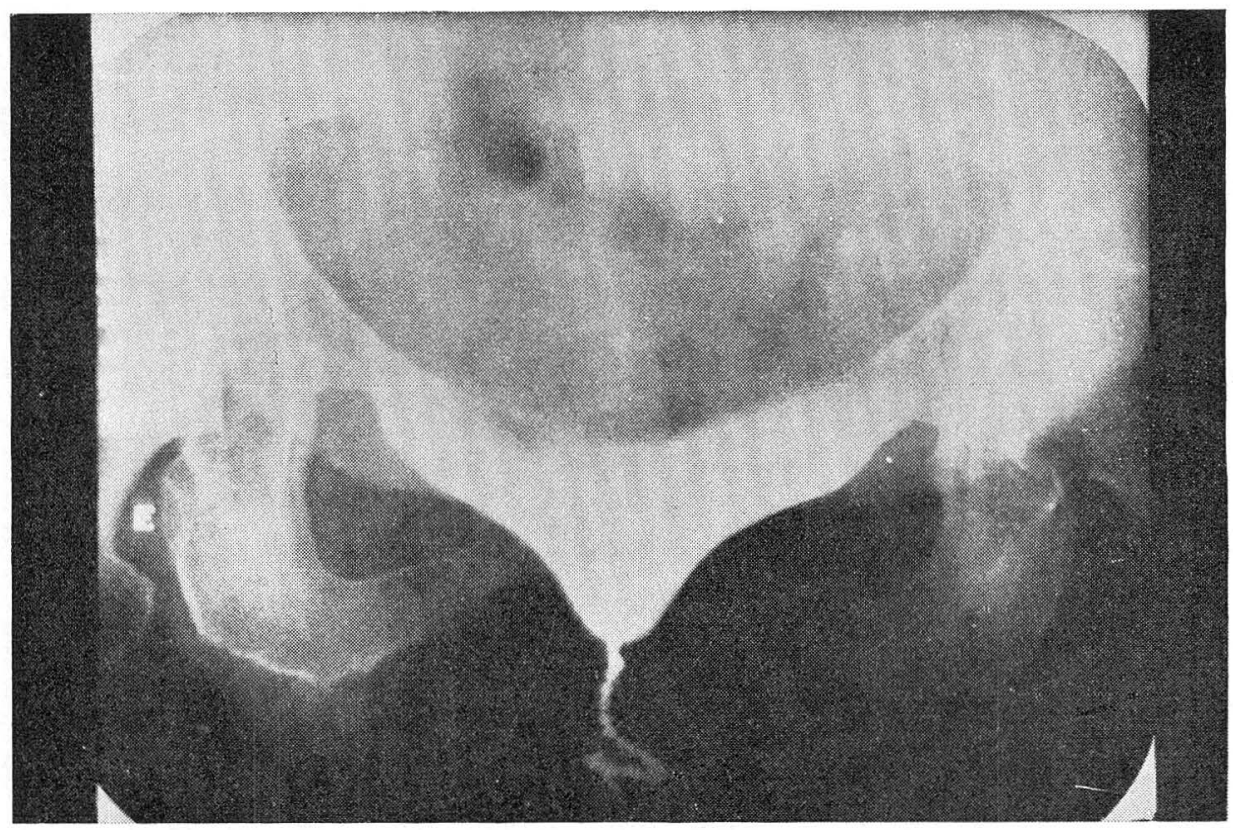

FIGURA 5

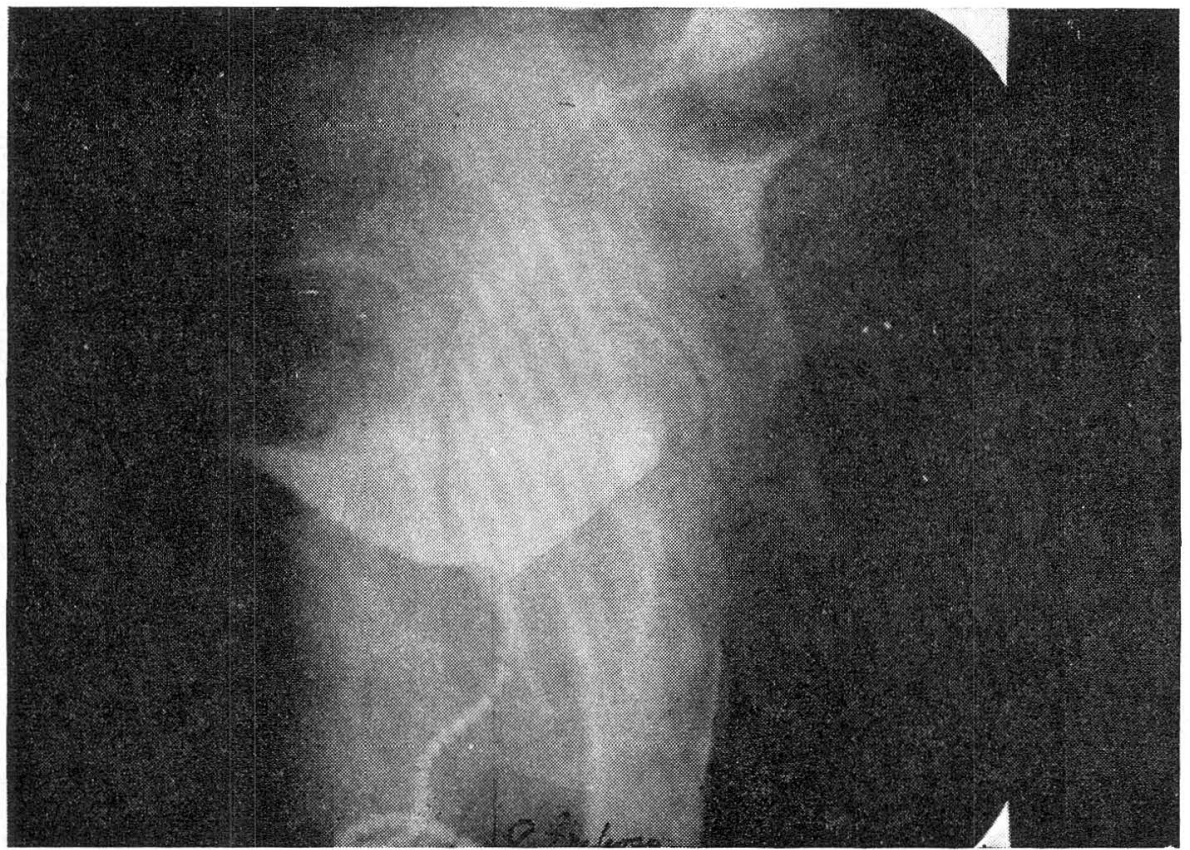

FIGURA 6 


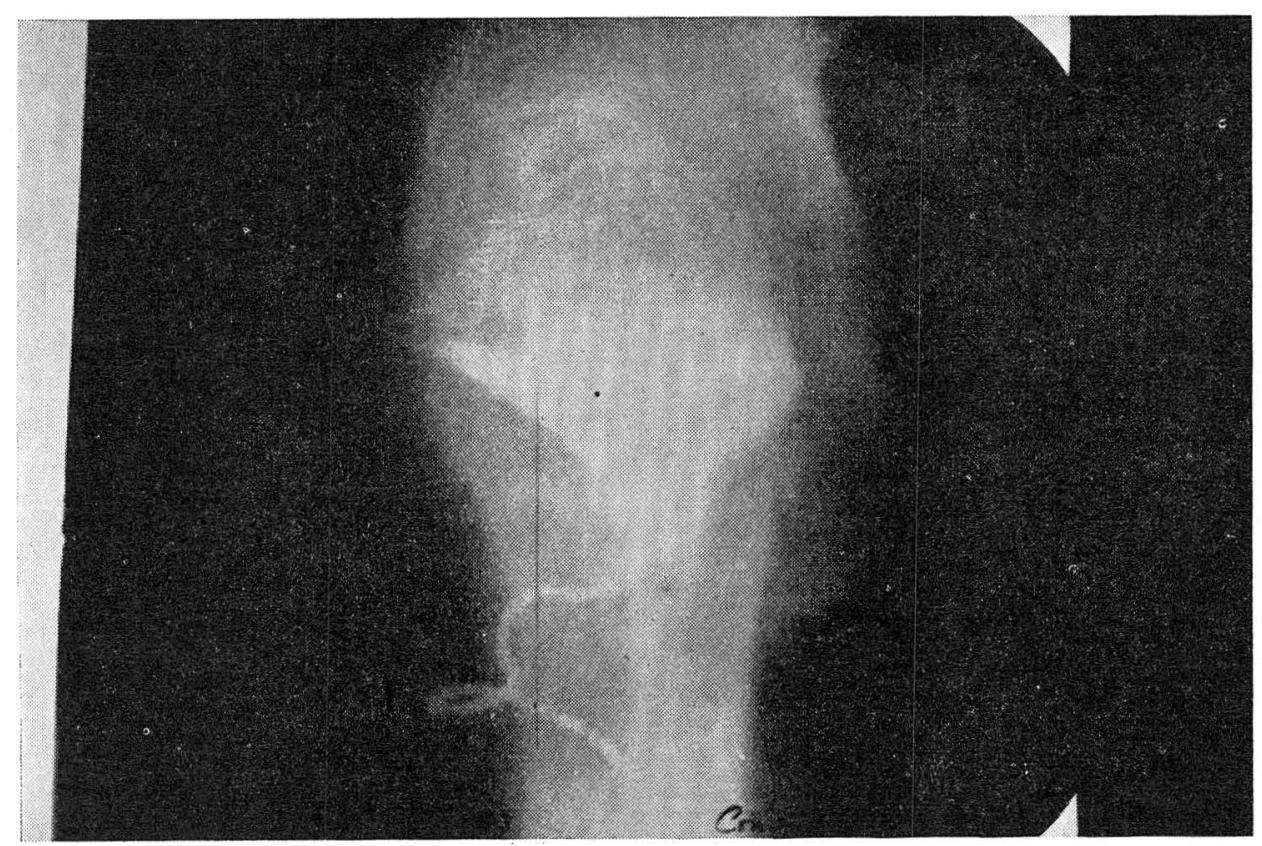

FIGURA 7

8. Uretrocistometría directa. Se le practicó a varias pacientes como parte de la evaluación post-operatoria. La técnica correspondiente y las posibilidades de este procedimiento, se describen en comunicación aparte (19).

Una vez que todos los estudios estaban hechos y que se consideraba a la paciente en condiciones adecuadas para una intervención quirúrgica, el grupo estudiaba la historia clínica completa y se acordaba el procedimiento que se le habría de practicar. Se clasificaron los casos según la pauta trazada por Green (12) y se definió la conducta siguiendo sus mismas orientaciones, así :

Tipo 1: 1) Plastias vaginales (colporrafia anterior y posterior a menudo con histerectomía vaginal), si el problema en conjunto indicaba la vía vaginal.

2) Suspensión de Marshall-Marchetti con histerectomía abdominal y perineorrafia, cuando estaba indicada, en situaciones en las cuales el problema se resolvía mejor por vía abdominal.

Tipo 11: 1) Si estaba indicada la vía vaginal debido a prolapso asociado y relajación perineal, se hacía histerectomía vaginal con colporrafia anterior y posterior, junto con una suspensión de Marshall-Marchetti, que se ejecutaba inmediatamente después de terminar el procedimiento vaginal.

2) Si no era evidente la necesidad de una reparación vaginal, se hacía una suspensión de Marshall-Marchetti de entrada, ejecutando primero 
una histerectomía abdominal a través de la misma incisión.

3. Para la falla ocasional que ocurría después de una suspensión de Marshall-Marchetti, se hacía la intervención con tira de fascia del recto.

\section{Estudio clínico}

Ingresaron originalmente al estudio un total de 37 pacientes. De estas se excluyeron 9 debido a que no se demostró que sufrieran incontinencia de esfuerzo. Esto dejó un total de 28 pacientes.
Rev. Col. Obst. y Ginec.

Al analizar las historias, se observa que hay antecedentes quirúrgicos ginecológicos en 5 pacientes. La patología ginecológica asociada se describe en el Cuadro 1, en el cual puede observarse que la mayoría de las pacientes, 22 de las 28, tenían algún trastorno ginecológico susceptible de corrección quirúrgica; de éstas, 16, es decir, un poco más de la mitad del total de las pacientes incluídas en el estudio, tenían patología ginecológica que incluía trastorno anatómico del tracto urinario inferior (Figuras 10, $11)$.

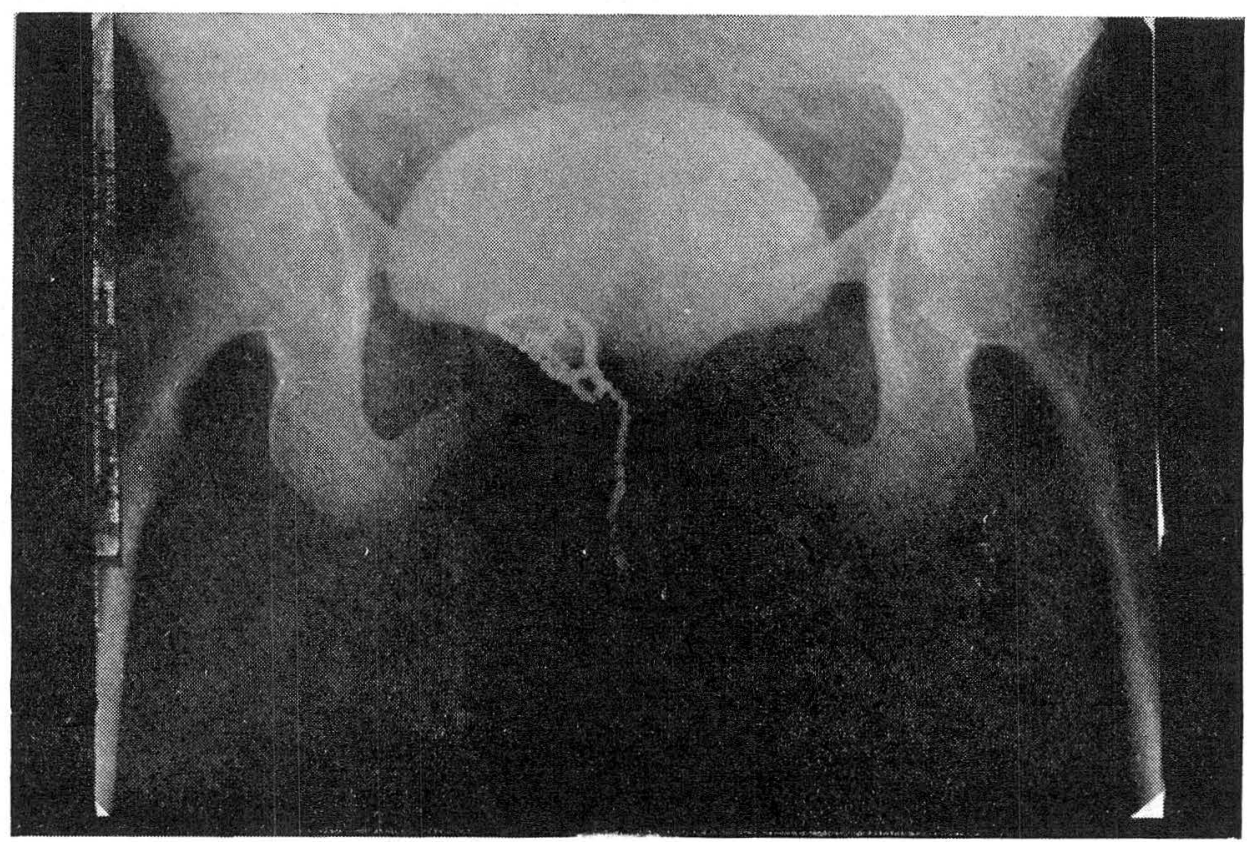

FIGURA 10 


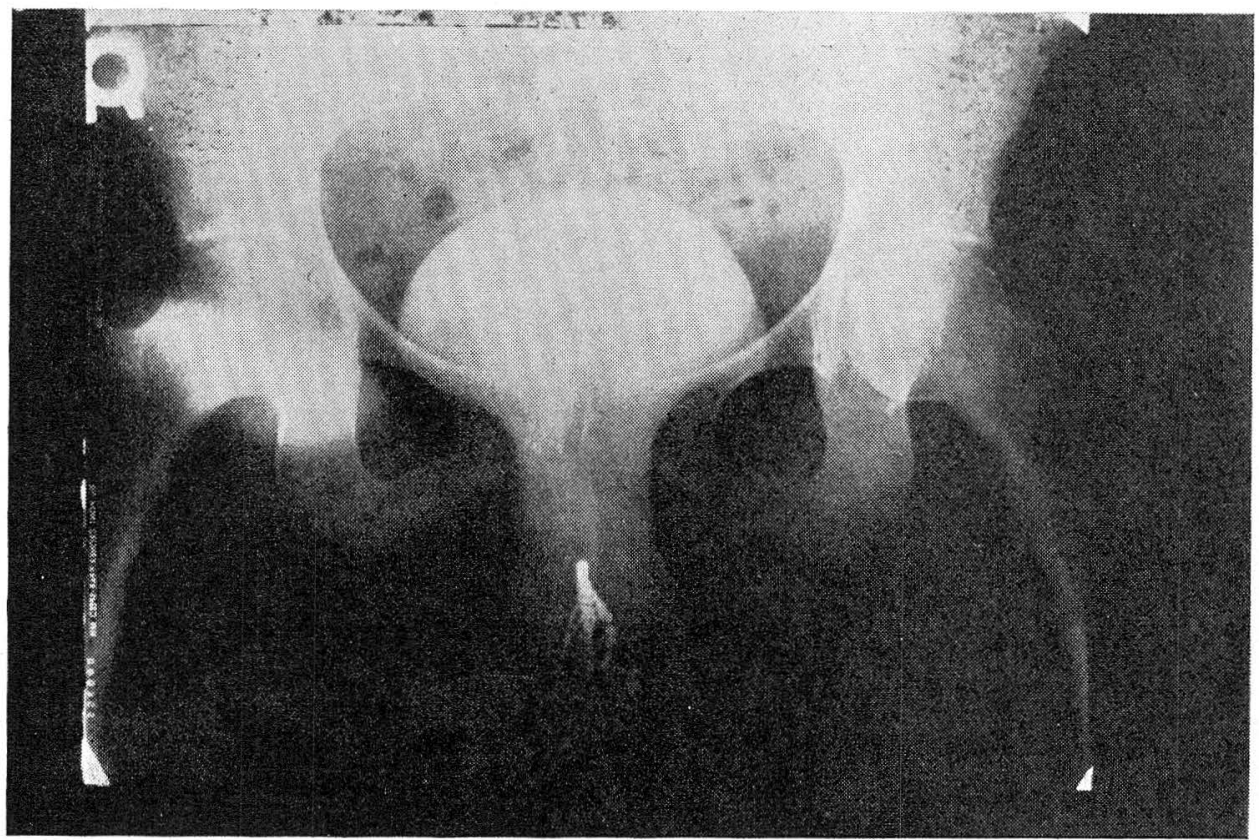

FIGURA 11

\section{CUADRO 1}

PATOLOGIA GINECOLOGICA ASOCIADA EN 28 PACIENTES CON INCONTINENCIA URINARIA DE ESFUERZO. - HOSPITAL UNIVERSITARIO SAN VICENTE DE PAUL. - MEDELLIN.

P. N. Cardona y otros, 1969.

Patología asociada

Número de Casos

Prolapso genital integral de grados II y III

Rectocele y desgarro perineal

Prolapso genital de I grado

Periné incontinente

Cistocele de I grado

Ninguna patología

TOTAL

La encuesta se realizó en un total de 22 de las 28 pacientes, con los resultados que se aprecian en el Cuadro 2.

Las pruebas de Bonney y Read fueron positivas en todos los casos en que se levaron a cabo, que fueron 23. No se hicieron en 5 casos.
Los resultados del estudio radiológico se aprecian claramente en el Cuadro 3.

En 5 casos se hizo cistometrograma; en ninguno de ellos se encontró vejiga neurogénica. Llamó la atención la gran capacidad vesical de todas las pacientes. 


\section{CUADRO 2}

RESULTADOS DE LA ENCUESTA SOBRE CARACTERISTICAS DE LA INCONTINENCIA URINARIA EN 28 PACIENTES. - HOSPITAL UNIVERSITARIO SAN VICENTE DE PAUL. - MEDELLIN.

P. N. Cardona y otros, 1969.

Resultados de la encuesta

Número de Casos

La encuesta corrobora los resultados del examen

La encuesta no corrobora los resultados del examen

La encuesta no contribuye a explicar la patología

No se hizo encuesta

TOTAL

\section{CUADRO 3}

RESULTADOS DE LA URETROCISTOGRAFIA CON CADENA EN 28 PACIENTES CON INCONTINENCIA URINARIA DE ESFUERZO. - HOSPITAL UNIVERSITARIO SAN VICENTE DE PAUL. - MEDELLIN.

P. N. Cardona y otros, 1969.

Resultados de la uretrocistografía

Número de Casos

Uretrocistografía muestra incontinencia tipo I

Uretrocistografía muestra incontinencia tipo II

Uretrocistografía normal

Uretrocistografía no satisfactoria

No se hizo uretrocistografía

TOTAL

\section{CUADRO 4}

CLASE DE TRATAMIENTO Y RESULTADOS EN 6 PACIENTES CON DIFERENTE PATOLOGIA GINECO. LOGICA QUE PRESENTABAN UN CUADRO RADIOLOGICO TIPO I DE INCONTINENCIA URINARIA DE ESFUERZO. - HOSPITAL UNIVERSITARIO SAN VICENTE DE PAUL. - MEDELLIN.

P. N. Cardona y otros, 1969.

\begin{tabular}{lcccc}
\hline Clase de patología ginecológica & $\mathbf{N}^{0}$ de casos & Tipo de Operación & Resultado & $\begin{array}{c}\text { Meses de } \\
\text { seguimiento }\end{array}$ \\
\hline Prolapso de II grado & 2 & 1 : Plastias & ? & 0 \\
Cistocele de I grado & 1 & $2:$ M. M. (1) & Bueno & 2 \\
Desgarro perineal & 1 & M. M. & Bueno & 2 \\
& & M. M., H. A. & Malo & 2 \\
Rectocele & 1 & (2) & Bueno & 4 \\
Ninguna & 1 & M. M. & Pueno & 2 \\
\hline
\end{tabular}

Notas: (1) Marshall-Marchetti.

(2) Histerectomía abdominal. 
En 23 casos se hizo estudio bacteriológico. En 20 fue negativo y en 3 fue positivo para algún tipo de germen. En todos ellos se hizo tratamiento adecuado previo a la intervención.

Los resultados del tratamiento se aprecian en los cuadros, que siguen, y se discriminan de acuerdo a los ha- llazgos de la uretrocistografía, principal criterio para la selección del mismo.

En 5 de 6 casos con incontinencia tipo I, que fueron tratados por diferentes métodos, el resultado fue bueno aunque el seguimiento en general es muy corto para conocer la eficacia final del tratamiento (Cuadro 4).

\section{CUADRO 5}

CLASE DE TRATAMIENTO $Y$ RESULTADOS EN 10 PACIENTES CON DIFERENTE PATOLOGIA GINECOLOGICA, QUE PRESENTABAN UN CUADRO RADIOLOGICO TIPO II DE INCONTINENCIA URINARIA DE ESFUERZO. - HOSPITAL UNIVERSITARIO SAN VICENTE DE PAUL. - MEDELLIN. P. N. Cardona y otros, 1969.

\begin{tabular}{|c|c|c|c|c|c|}
\hline Clase de patología ginecológica & $\mathrm{N}^{0}$ de casos & Tipo & de Operación & Resultado & $\begin{array}{l}\text { Meses de } \\
\text { seguimiento }\end{array}$ \\
\hline Prolapso de III grado & 2 & $\begin{array}{l}\text { 1: } \\
2:\end{array}$ & $\begin{array}{l}\text { H. V. (1) } \\
\text { M., M. M. } \\
\text { (2) }\end{array}$ & $\begin{array}{c}? \\
\text { Bueno }\end{array}$ & $\begin{array}{l}2 \\
7\end{array}$ \\
\hline Prolapso de II grado & 3 & $\begin{array}{l}1: \\
2: \\
3:\end{array}$ & $\begin{array}{l}\text { P. K. (3) } \\
\text { H. A., B. } \\
\text { y P. (4) } \\
\text { P. K. }\end{array}$ & $\begin{array}{l}\text { Bueno } \\
\text { Bueno } \\
\text { Bueno }\end{array}$ & 2 \\
\hline Prolapso de I grado & 3 & $\begin{array}{l}1: \\
2: \\
3:\end{array}$ & $\begin{array}{l}\text { Ball } \\
\text { Plastias } \\
\text { M. M. }\end{array}$ & $\begin{array}{l}\text { Bueno } \\
\text { Bueno } \\
\text { Malo }\end{array}$ & $\begin{array}{r}10 \\
1 \\
4\end{array}$ \\
\hline $\begin{array}{l}\text { Histerocele } \\
\text { Rectocele }\end{array}$ & $\begin{array}{l}1 \\
1\end{array}$ & & $\begin{array}{l}\text { H. V., M. M. } \\
\text { Igual }\end{array}$ & $\begin{array}{l}\text { Bueno } \\
\text { Malo }\end{array}$ & $\begin{array}{l}7 \\
6\end{array}$ \\
\hline
\end{tabular}

Notas: (1) Histerectomía vaginal.

(2) Mánchester y Marshall-Marchetti.

(3) Plastias y Kelly.

(4) Histerectomía abdominal, Ball y Plastias.

\section{CUADRO 6}

CLASE DE TRATAMIENTO Y RESULTADOS EN 7 PACIENTES CON DIFERENTE PATOLOGIA GINECOLOGICA, SIN ESTUDIO RADIOLOGICO PREVIO A LA INTERVENCION. HOSPITAL UNIVERSITARIO SAN VICENTE DE PAUL. - MEDELLIN.

P. N. Cardona y otros, 1969.

\begin{tabular}{|c|c|c|c|c|c|}
\hline Clase de patología ginecológica & $N^{0}$ de casos & Tipo & de Operación & Resultado & $\begin{array}{l}\text { Meses de } \\
\text { seguimiento }\end{array}$ \\
\hline Prolapso de III grado & 2 & & $\begin{array}{l}\text { H. V. } \\
\text { P. K. }\end{array}$ & $\begin{array}{l}\text { Malo } \\
\text { Bueno }\end{array}$ & $\begin{array}{l}5 \\
4\end{array}$ \\
\hline Prolapso de II grado & 1 & & $\begin{array}{l}\text { P. K. } \\
\text { M. } M .\end{array}$ & Malo & 2 \\
\hline Rectocele de II grado & 1 & & $\begin{array}{l}\text { H. A. } \\
\text { M. M. }\end{array}$ & Bueno & 3 \\
\hline $\begin{array}{l}\text { Periné incontinente } \\
\text { Ninguna }\end{array}$ & $\begin{array}{l}1 \\
2\end{array}$ & $\begin{array}{l}1: \\
2:\end{array}$ & $\begin{array}{ll}M . & M \\
M . & M \\
M . & M\end{array}$ & $\begin{array}{l}\text { Malo } \\
\text { Bueno } \\
\text { Bueno }\end{array}$ & $\begin{array}{l}4 \\
4 \\
3\end{array}$ \\
\hline
\end{tabular}


El procedimiento empleado en la mayoría de ellos fue Marshall-Marchetti.

En los casos con incontinencia tipo II, (Cuadro 5) hubo 2 en los cuales el resultado fue malo después de practicarles un Marshall-Marchetti. Es posible que haya faltado una mejor evaluación diagnóstica en estas pacientes antes de intervenirlas, con lo cual posiblemente se hubiera escogido un procedimiento terapéutico que les hubiera sido de mayor valor.

Como se aprecia en el mismo cuadro, en 7 casos que presentaban un cambio radiológico tipo II de incontinencia de esfuerzo, el resultado fue bueno aunque no se han seguido por un tiempo lo suficientemente largo para permitir sacar ninguna conclusión. En 7 casos más no se hizo ningún estudio radiológico previo a la intervención quirúrgica y en 3 de ellos el resultado fue malo. Esto puede dar una idea de lo necesario que es practicar tal estudio antes de emprender el tratamiento quirúrgico de la entidad (Cuadro 6).

En una paciente en la cual la uretrocistografía fue normal y que tenía un cisto y rectocele de I grado, se practicó un Marshall-Marchetti y durante el seguimiento, que fue de tres meses, el resultado fue malo.

En cuatro casos la uretrocistografía fue poco satisfactoria, por lo cual no sirvió como base para establecer el tratamiento. El cuadro 7 muestra el que se efectuó en cada caso, y los resultados correspondientes.

Si se suman los casos en los que no se hizo uretrocistografía, con

\section{CUADRO 7}

CLASE DE TRATAMIENTO Y RESULTADOS EN 4 PACIENTES CON INCONTINENCIA URINARIA DE ESFUERZO Y DIFERENTE PATOLOGIA GINECOLOGICA, EN LAS CUALES EL ESTUDIO RADIOLOGICO FUE INSATISFACTORIO. - HOSPITAL UNIVERSITARIO SAN VICENTE DE PAUL. - MEDELLIN. P. N. Cardona y otros, 1969.

\begin{tabular}{lcccc}
\hline Clase de patología ginecológica & No de casos & Tipo de Operación & Resultado & $\begin{array}{c}\text { Meses de } \\
\text { seguimiento }\end{array}$ \\
\hline Relajación perineal & 1 & M. M. & Bueno & 10 \\
Ninguna patología & 1 & M. M. & Bueno & 12 \\
& 1 & M. M. & Bueno & 4 \\
\hline
\end{tabular}

\section{CUADRO 8}

TIPO DE CURSO POST-OPERATORIO EN 28 PACIENTES INTERVENIDAS PARA INCONTINENCIA URINARIA DE ESFUERZO, SEGUN EL RESULTADO.

HOSPITAL UNIVERSITARIO SAN VICENTE DE PAUL. - MEDELLIN.

P. N. Cardona y otros, 1969.

Curso post-operatorio

\begin{tabular}{lcrr}
\hline Resultado del tratamiento & Febril & Afebril & Totales \\
\hline Bueno & 6 & 12 & 18 \\
Malo & 5 & 4 & 9 \\
Desconocido & 11 & 1 & 1 \\
$\quad$ TOTALES & 11 & 17 & 28 \\
\hline
\end{tabular}


aquellos en que esta fue poco satisfactoria, se ve que en 7 de 11 el resultado fue bueno. El tipo de curso post-operatorio parece que tiene que ver con los resultados de la operación, pues de 18 casos en que el resultado fue bueno, en 12 el curso fue afebril, mientras que en los ca- sos en que el resultado fue malo, en cinco de nueve del curso fue febril. (Cuadro 8).

Las complicaciones que ocurrieron se discriminan en el cuadro 9, que muestra la época de su aparición de acuerdo con la intervención.

\section{CUADRO 9}

INCIDENCIA DE COMPLICACIONES OCURRIDAS EN 28 PACIENTES INTERVENIDAS PARA INCONTINENCIA URINARIA DE ESFUERZO, DE ACUERDO AL TIPO Y A LA EPOCA DE SU APARICION. HOSPITAL UNIVERSITARIO SAN VICENTE DE PAUL. - MEDELLIN.

P. N. Cardona y otros, 1969.

Complicaciones

Número de Pacientes

\section{Inmediatas}

Shock post-operatorio e isquemia sub-endocárdica

Supuración de la herida quirúrgica

Tardías

Fístula vésico-vaginal

Ninguna complicación TOTAL

Como se puede observar, una de estas complicaciones fue de tipo urinario y es posible que se hubiera podido obviar si se hubiera hecho un mejor estudio urológico previo a la intervención y se hubiera excluído cualquier patología asociada que pudiera existir.

El control radiológico post-operatorio se hizo solamente en una paciente. En él se observó que había pasado de un tipo I de incontinencia a una imagen de apariencia normal (Figuras 14, 15, 16, 17). Se hicieron 12 estudios de uretrocistometría directa, todos ellos en pacientes que ya habían sido intervenidas. Los detalles de este procedimiento aparecen en otra comunicación (19).

\section{Discusión}

El interés por el estudio de la incontinencia urinaria de esfuerzo en Colombia tiene poca tradición, a juzgar por las escasas publicaciones que pueden encontrarse al revisar los índices de la literatura médica nacional. Sin embargo, los estudios más recientes llevados a cabo en otros países, especialmente por Bailey, Jeffcoate, Hodgkinson y Green, han conducido al establecimiento de una serie de conocimientos básicos de la fisiopatología y la anatomía patológica de la incontinencia de esfuerzo, lo cual a su vez ha permitido sentar bases más racionales de tratamiento que han significado una mejoría apreciable en los resultados, como puede apreciarse por el porcentaje de $96 \%$ de curaciones entre los 4 y 10 años después del tratamiento, que han obtenido algunos autores (13).

El conocimiento de estos hechos, aunado a otro no menos importante, cual es el de que la incidencia de la incontinencia urinaria de esfuerzo en las pacientes que acuden a la consulta externa de entidades hospitalarias de nuestra población alcanza ci- 
Marzo-Abril 1970

132 PEDRO NEL CARDONA y cols. Rev. Col. Obst. y Ginec.

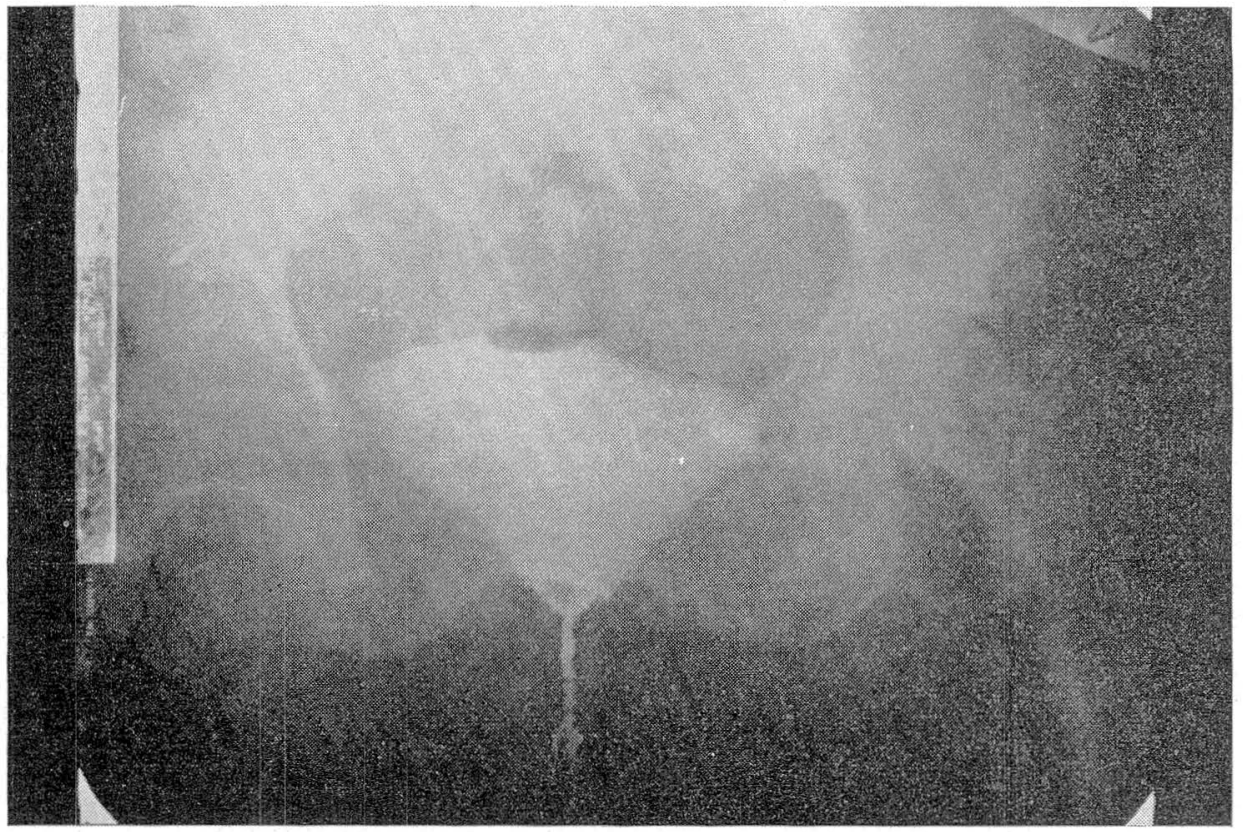

FIGURA 14

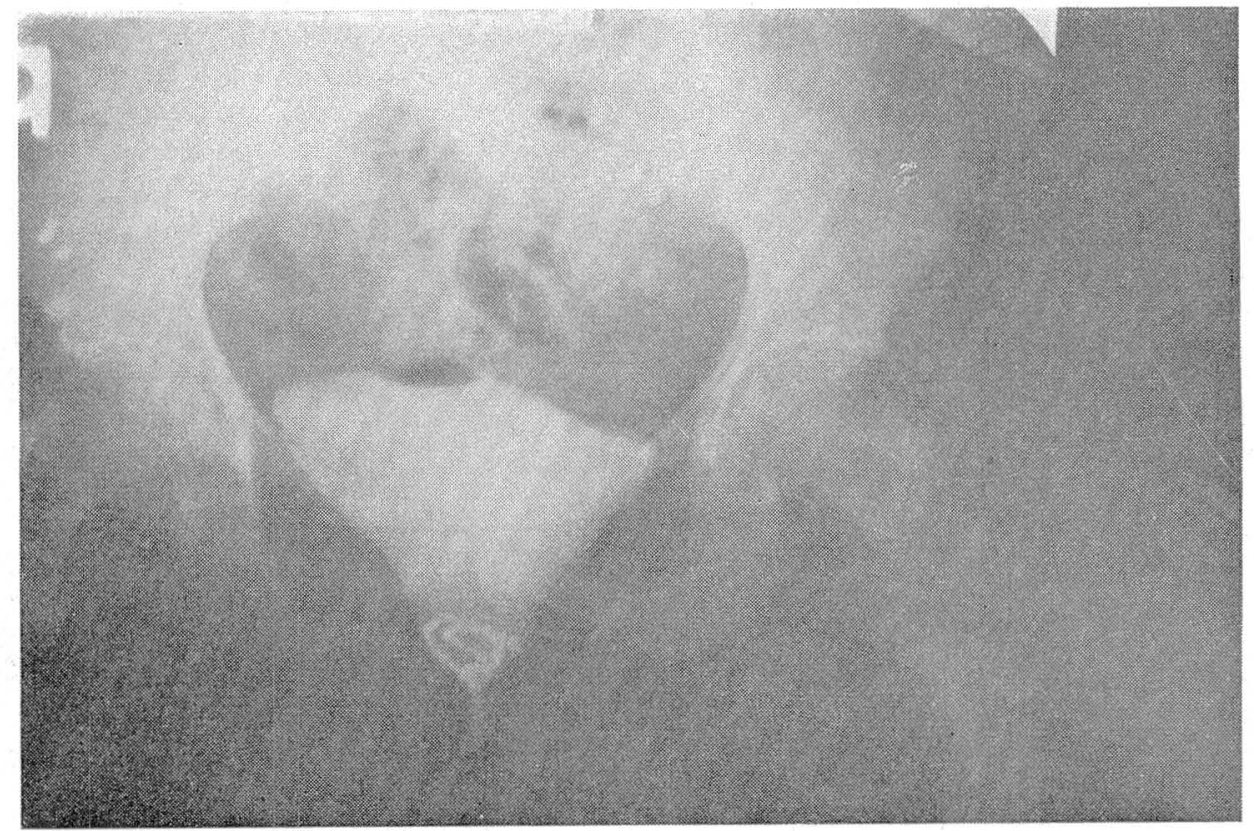

FIGURA 15 
Vol. $X X 1$

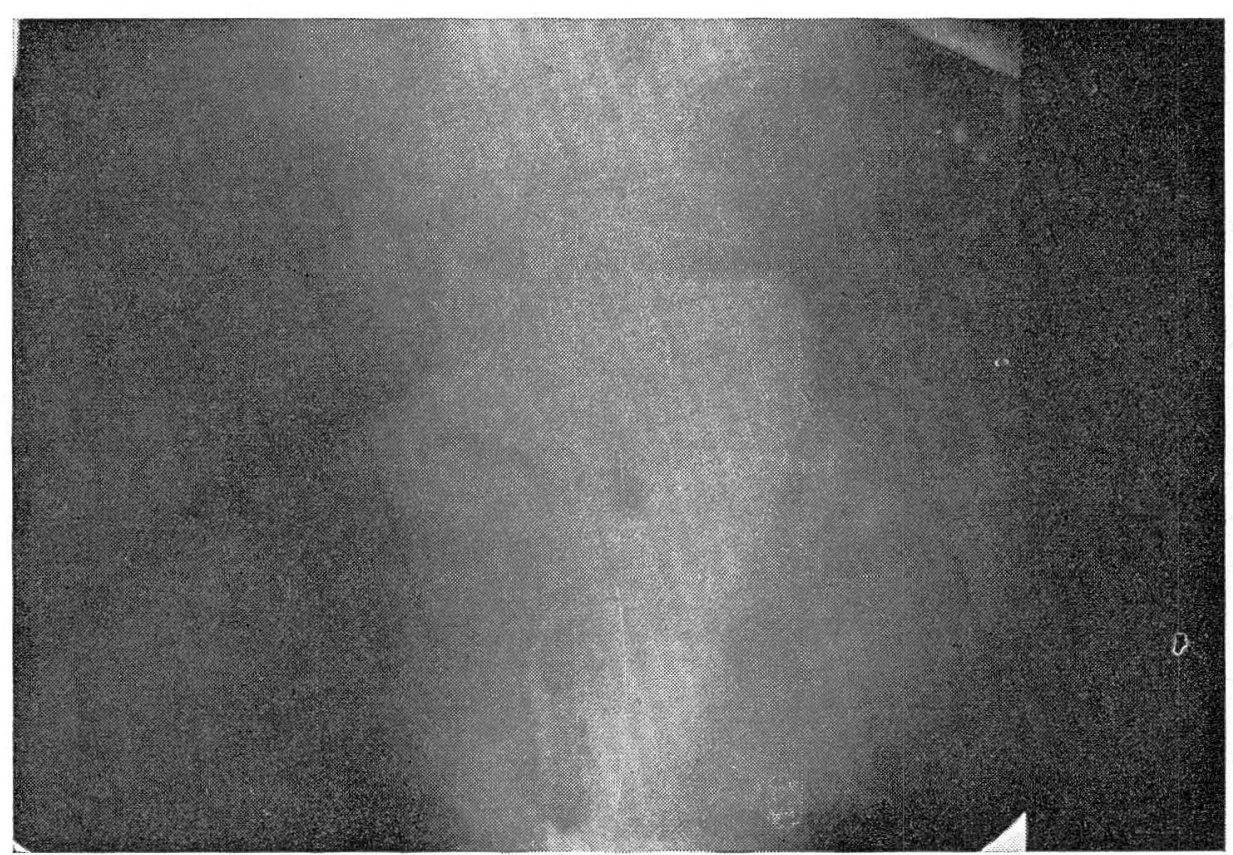

FIGURA 16

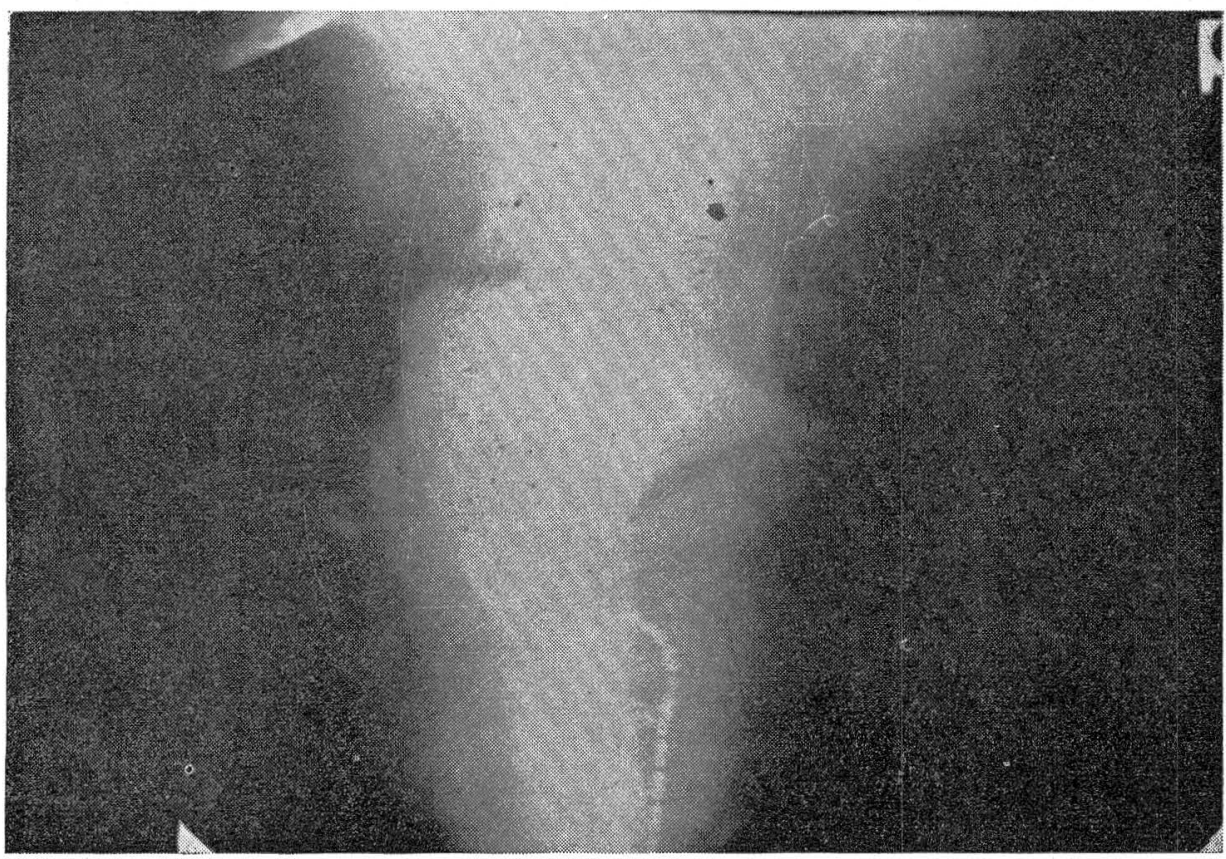

FIGURA 17 
fras considerables como lo demuestra la encuesta realizada por dos de los autores (20), nos movió a establecer una pauta definida para el estudio y tratamiento de tales pacientes, siguiendo las orientaciones de los autores mencionados, y particularmente las de Green (12) y constituyendo un grupo de estudio que se encargaría del manejo total de dichas pacientes y de su seguimiento por un tiempo lo suficientemente largo que permitiera sacar conclusiones. Este grupo continúa estudiando tales pacientes y otras que se han ido agregando, y los resultados de su experiencia serán conocidos periódicamente.

En el presente trabajo publicamos los resultados de nuestra experiencia en los primeros 28 casos tratados, siguiendo las orientaciones mencionadas. Tal como puede observarse, aún ha transcurrido un tiempo muy corto desde el momento del tratamiento $y$ los resultados no tienen aún un valor significativo. Por otra parte, el grupo de pacientes es pequeño y su fragmentación en subgrupos no permite sacar conclusiones valederas. Se observa sin embargo que la orientación general dá la sensación de ser adecuada a juzgar por los resultados iniciales del tratamiento aplicado a las distintas pacientes.

\section{Conclusiones}

Todas son naturalmente preliminares pero aún así creemos que pueden tener valor para dar una impresión general sobre esta entidad y su tratamiento. Las más salientes nos parecen las siguientes:

1. La existencia de patología ginecológica asociada, que estuvo presente en 22 de las 28 pacientes.

2. La encuesta sobre características de la incontinencia confirmó el diagnóstico en la gran mayoría de los casos.

3. Aún es muy poca nuestra experiencia con la uretrocistografía en cadena, pero está de acuerdo con la de los demás autores en que es el principal método de que se dispone para establecer en forma objetiva el diagnóstico y para escoger el tratamiento que mejor conviene a una paciente dada. Es posible que solamente baste con tomar dos placas laterales, una en reposo y la otra pujando, pues con ellas es con las que se obtienen los datos más importantes.

4. Se destaca la importancia de un estudio urológico completo, sobre todo en las pacientes que presentan infección urinaria, obstrucción u otra patología evidente, así como en aquellas que hayan tenido fracasos con tratamientos previos. La colaboración del urólogo en el estudio y tratamiento de los casos, es imprescindible.

5. Los grados 1 de incontinencia urinaria de esfuerzo diagnosticados con el estudio radiológico, parecen responder bien a la intervención de Marshall-Marchetti.

6. En los grados II se han realizado procedimientos variados, algunos no muy ajustados a la pauta que se adoptó originalmente, con resultado bueno en 7 de los 10 casos.

7. En casi dos tercios de los casos sin estudio radiológico o con estudio poco satisfactorio, el resultado fue bueno. Este hecho es inexplicable en el momento actual, y debe verificarse si persiste cuando el número de casos que se haya acumulado tenga validez estadística.

\section{Resumen}

Se presenta un corto recuento de los hallazgos recientes sobre la fisio- 
patología y la anatomía patológica de la incontinencia urinaria de esfuerzo en la mujer, y se refiere la manera como se organizó el estudio y tratamiento de pacientes que consultaron por esa queja en el Hospital Universitario San Vicente de Paúl, en Medellín, siguiendo las pautas establecidas por Green. Se presentan los resultados preliminares de las primeras 28 pacientes tratadas siguiendo tales pautas.

\section{Summary}

A short account of the most recent findings in regard to the physiopathology and the anatomic derangement present in cases of urinary stress incontinence is presented.

The way in which a systematic study of these patients was undertaken at the Hospital Universitario San Vicente de Paúl in Medellin Colombia, following the orientations given gy Green, is shown.

The preliminary results of the first 28 patients so treated are presented and discused.

\section{Agradecimientos}

Agradecemos a los Dres. Luis Fernando Uribe B., Daniel Correa, del Departamento de Rayos $X, y$ al personal de técnicas del mismo Departamento, por la colaboración que nos prestaron durante la realización del presente trabajo.

\section{BIBLIOGRAFIA}

1 DURPHEE, R. B.: The anterior vaginal suspension operation. J. Internat. Coll. Surg. 30 (6): 791, Dec. 1958.

2 FRANKEL, D. S.: Full-thickness anterior colporrhaphy for correction of urinary stress incontinence. Amer. J. Obstet. \& Gynec. 76 (6) : 1185, Dec. 1958.

3 GARDINER, S. H.: Vaginal surgery for stress incontinence. Clin. Obstet. \& Gynec. 6 (1): 178, Mar. 1963.
4 WHARTON, L. R. \& TE LINDE, R. W.: An evaluation of fascial sling operation for urinary incontinence in female patients. J. Urol. 82 (1): 76, Jul. 1959.

5 ZACHARIN, R. F. \& GLEADELL, L. W.: Abdomino-perineal urethral suspension. Amer. J. Obstet. \& Gynec. 86 (8): 981, Aug. 15, 1963.

6 MARCHETTI, A. A., MARSHALL, V. F. \& SHULTIS, L. D.: Simple vesicourethral suspension. A. Survey. Amer. J. Obstet. \& Gy. nec. 74 (1): 57, 1957.

7 CHASSAR MOIR, J.: Tha gauze hammock operation. J. Obstet. Kynaec. Brit. Cwlth. 75 (1): 1, Jan. 1968.

8 BAILEL, K. V.: A clinical investigation into uterine prolapse with stress incontinence. Treatment by modified Manchester colporrhaphy. J. Obstet. Gynaec. Brit. Emp. Part I, 61: 291, 1954. Part 11, 63: 663, 1956 and Part III, 79; 947, 1963.

9 JEFFCOATE, T. N. A.: The principles governing the treatment of stress incontinence of urine in the female. Brit. J. Urol. 37 (6): 633, Dec. 1965.

10 HODGINSON, C. P. Urinary stress incontinence in the female. Clin. Obstet. \& Gynec. 6 (1): 154, Mar. 1963.

11 HODGKINSON, C. P.: Stress urinary incontinence in the female. Surg. Gynec. \& Obstet. 120 (3): 505, Mar. 1965.

12 GREEN, Th. H.: Development of a plan for the diagnosis and treatment of urinary stress incontinence. Amer. J. Obstet. \& Gynec. 83 (5): 632, Mar. 1962.

13 GREEN, Th. H.: The problem of urinary stress incontinence in the female. Obstet. \& Gynec. Survey. 23: 603, 1968.

14 BELTRAN, A.: Incontinencia urinaria de esfuerzo en la mujer. Rev. Colombia Obstet. \& Gynec. 12 (2): 108, 1961.

15 BERNAL, E.: La uretro-vésico-suspensión de Marshall-Marchetti-Krantz en la corrección de la incontinencia urinaria de esfuerzo. Rev. Colombia Obstet. \& Ginec. 15: 159, 1964

16 LUND, C. J.: Types of urinary incontinence. Clin. Obstet. \& Gynec. 6 (1): 125, 1963.

17 MITCHELL, G. W.: Cystometry in the evaluation of urinary incontinence. Clin. Obstet. \& Gynec. 1 (3): 678, 1958.

18 Urethrocystograms. Metallic bead chain technique. Clin. Obstet. \& Gynec. 1 (3): 668, 1958. 
19 JUBIZ, A. y BOTERO J.: La uretrocistometría directa en el estudio de la función vesical. Presentado al $8^{\circ}$ Congreso Colombiano de Obstetricia y Ginecología, Manizales, Colombia, Dic. 2-6, 1969.
20 BOTERO, J. y JUBIZ, A.: Incidencia de la incontinencia urinaria de esfuerzo en la mujer. Presentado al $8^{\circ}$ Congreso Colombiano de Obstetricia y Ginecología, Manizales, Colombia, Dic. 2-6, 1969. 